\title{
The role of the JAK-STAT pathway in neural stem cells, neural progenitor cells and reactive astrocytes after spinal cord injury (Review)
}

\author{
TIANYI WANG ${ }^{1,2^{*}}$, WENQI YUAN ${ }^{1 *},{\text { YONG } \text { LIU }^{1 *}, \text { YANJUN ZHANG }^{3}, \text { ZHIJIE WANG }^{4}, \text { XIANHU ZHOU }}^{1}$, \\ GUANGZHI NING ${ }^{1}$, LIANG ZHANG ${ }^{5}$, LIWEI YAO ${ }^{1}$, SHIQING FENG ${ }^{1}$ and XIAOHONG KONG ${ }^{6}$ \\ ${ }^{1}$ Department of Orthopedics, Tianjin Medical University General Hospital, Tianjin 300052; ${ }^{2}$ Department of Orthopedics, \\ The 266th Hospital of the Chinese People's Liberation Army, Chengde, Hebei 067000; ${ }^{3}$ Department of Orthopedics, \\ Capital Medical University Luhe Hospital, Beijing 100000; ${ }^{4}$ Department of Paediatric Internal Medicine, Affiliated Hospital \\ of Chengde Medical College, Chengde, Hebei 067000; ${ }^{5}$ Department of Orthopedics, The Second Hospital \\ of Tianjin Medical University, Tianjin 300211; ${ }^{6}$ School of Medicine, Nankai University, Tianjin 300071, P.R. China
}

Received September 24, 2014; Accepted October 16, 2014

DOI: 10.3892/br.2014.401

\begin{abstract}
Patients with spinal cord injuries can develop severe neurological damage and dysfunction, which is not only induced by primary but also by secondary injuries. As an evolutionarily conserved pathway of eukaryotes, the JAK-STAT pathway is associated with cell growth, survival, development and differentiation; activation of the JAK-STAT pathway has been previously reported in central nervous system injury. The JAK-STAT pathway is directly associated with neurogenesis and glia scar formation in the injury region. Following injury of the axon, the overexpression and activation of STAT3 is exhibited specifically in protecting neurons. To investigate the role of the JAK-STAT pathway in neuroprotection, we summarized the effect of JAK-STAT pathway in the following three sections: Firstly, the modulation of JAK-STAT pathway in proliferation and differentiation of neural stem cells and neural progenitor cells is discussed; secondly, the time-dependent effect of JAK-STAT pathway in reactive astrocytes to reveal their capability of neuroprotection is revealed and lastly, we focus on how the astrocyte-secretory polypeptides (astrocyte-derived cytokines and trophic factors) accomplish neuroprotection via the JAK-STAT pathway.
\end{abstract}

Correspondence to: Professor Shiqing Feng, Department of Orthopedics, Tianjin Medical University General Hospital, 154 Anshan Road, Heping District, Tianjin 300052, P.R. China E-mail: professorfengsq@163.com

Professor Xiaohong Kong, School of Medicine, Nankai University, 94 Weijin Road, Nankai District, Tianjin 300071, P.R. China

E-mail: professorkxh@126.com

${ }^{*}$ Contributed equally

Key words: JAK-STAT pathway, astrocyte-secretory polypeptides, neural stem cells, neural progenitor cells, spinal cord injury, reactive astrocyte

\section{Contents}

1. Introduction

2. Modulation of the JAK-STAT pathway in proliferation and differentiation of neural stem cells and neural progenitor cells

3. Time-dependent effects of JAK-STAT pathway in reactive astrocytes

4. Astrocyte-secretory polypeptides promote neuroprotection via activation of the JAK-STAT pathway

5. Conclusion

\section{Introduction}

Every year $>10,000$ people in China are victims of spinal cord injury (SCI) due to traffic accidents, sports injuries and a number of other accidents (1). Patients with SCI may develop severe neurological damage and dysfunction $(1,2)$. Primary injury (mechanical injury) is the characteristic pathophysiology of acute SCI, which is followed by a phase of 'secondary injury' involving ischemia, calcium- and sodium-mediated cellular injury, cell death, inflammation and apoptosis $(3,4)$. As an evolutionarily conserved pathway of eukaryotes, the JAK-STAT pathway is associated with cell growth, survival, development and differentiation (5).

JAK-STAT is an intracellular signaling pathway that involves the activation of two families of proteins: The Janus kinases (JAK) and the signal transducer and activator of transcriptions (STAT). JAK is a class of four cytoplasmic protein tyrosine kinases that includes JAK1, JAK2, JAK3 and TYK2 (6). The STAT family contains seven transcription factors: STAT1, STAT2, STAT3, STAT4, STAT5A, STAT5B and STAT6 (7). The JAK-STAT pathway is a highly regulated and efficient system which predominantly regulates gene expression (5). This pathway includes the activation of cell membrane receptors by polypeptides, such as growth factors, hormones, or cytokines, which induce the activation of JAK in cell membranes (8-11). 
Protein tyrosine phosphorylation is a significant biochemical mechanism, by which growth factors or cytokines regulate cellular processes. Initially, JAKs undergo tyrosine phosphorylation with cell membrane receptor binding $(12,13)$. Subsequently, phosphorylated JAKs activate STATs in the cytoplasm through tyrosine phosphorylation, leading to the dimerization of STATs $(9,12,14,15)$. The STAT dimers translocate to the nucleus, where they bind to specific cis-elements, followed by the transcription of various target genes $(9,16)$.

The activation of JAK and STAT has been observed in the motoneurons of rats $(17,18)$. Activation of the JAK-STAT pathway has been previously reported in central nervous system (CNS) injury (19-22). The JAK-STAT pathway is directly associated with neurogenesis and glia scar formation of the injury region. Following an injury of the axon, the overexpression and activation of STAT3 are induced specifically in protecting neurons (17). Other studies have reported activation of STAT3 in reactive astrocytes of damage regions $(19,23)$. In the intact spinal cord, STAT3 is localized predominantly in motoneurons and dendrite-like structures in the anterior horn (24).

This report reviews the following: i) Modulation of the JAK-STAT pathway in proliferation and differentiation of neural stem cells (NSCs) and neural progenitor cells (NPCs); ii) the time-dependent effect of JAK-STAT pathway in reactive astrocytes; and iii) astrocyte-secretory polypeptides promoting neuroprotection via the activation of the JAK-STAT pathway.

\section{Modulation of JAK-STAT pathway in proliferation and differentiation of NSCs and NPCs}

Following a SCI, endogenous NSCs and NPCs proliferate and migrate to the lesion region, where they differentiate exclusively into astrocytes $(25,26)$. Previous studies have confirmed the existence of NSCs and NPCs in the spinal cord, which has increased the possibility of the spinal cord having the capability to self-repair in response to injury or disease through the application of endogenous NSCs and NPCs $(27,28)$. Furthermore, recent studies have demonstrated that transplanted NSCs can replace the lost neurons and glia following SCI, as well as forming functional relays to reconnect the spinal cord through the lesion $(29,30)$. Additionally, the modulation of the JAK-STAT pathway has been revealed in the proliferation and differentiation of NSCs and NPCs $(31,32)$.

JAK-STAT pathway promotes astrogliogenesis. Bonni et al (33) were the first to report the role of the JAK-STAT pathway in glial differentiation. The authors validated, in cortical precursor embryo cells, that activation of the ciliary neurotrophic factor (CNTF) receptor subsequently activates JAK1, STAT1 and STAT3 and induces the differentiation of NSCs and NPCs into astrocytes. Several additional studies have also confirmed the role of STAT3 in glial differentiation $(34,35)$. Furthermore, glycoprotein 130 (gp130)-mediated signaling has been demonstrated to induce the astrocytic differentiation of NSCs and NPCs through the JAK-STAT pathway $(33,36)$. Sriram et al (37) also revealed that the gp130-mediated activation of STAT3 is vital in the induction of astrogliosis. Gp130 is a type of multichain receptor complex on the cell membrane. This complex includes the ligand binding receptor and non-ligand binding membrane glycoprotein, gp130, which have been shown to be significant in signal transduction of cytokines, such as the interleukin (IL)-6 family (38). These cytokines bind to multichain receptor complexes and induce the dimerization of gp130, followed by activation of JAK in cell membranes. This subsequently phosphorylates STAT3 at Tyr705, resulting in translocation to the nucleus. A number of articles have reported that prolactin (PRL) also allows proliferation and differentiation of astrocytes, partially via the phosphorylation of JAK2, STAT1 and STAT3 (39-45). DeVito et al (46-50) demonstrated that PRL can stimulate astrocyte growth and the expression of several cytokines in their early studies. Furthermore, the authors found that PRL stimulates the growth of astrocytes through increasing the phosphorylation of tyrosines in the inactivation loop of JAK2 and the subsequent phosphorylation of STAT1a, STAT5a and STAT5b (51). Another study showed that in conditional knockout mice, STAT3 knock-down inhibited astrogliogenesis (52).

Modulation of the JAK-STAT pathway can induce neuronal and oligodendrocytic differentiation. Cytokines such as interleukin (IL)-15 that are expressed by the adult NSC of CNS, activate STAT1, STAT3 and STAT5 via phosphorylation of JAK, and this activation can be blocked by JAK inhibitors $(53,54)$. A number of studies have demonstrated that inhibitory proteins of the JAK-STAT pathway are strongly associated with neuronal differentiation and neurite outgrowth. These inhibitory proteins, including suppressor of cytokine signaling (SOCS)2, SOCS3 and SOCS6 can negatively regulate the JAK-STAT pathway induced by factors including insulin-like growth factor- 1 and growth hormone $(\mathrm{GH})(55,56)$. The overexpression of SOCS2 in NSC suppresses GH-signaling and promotes neuronal differentiation, while neurogenesis is inhibited (56). Following SCI, in adult mice, the absence of SOCS3 promotes gp130-mediated CNTF signaling via the JAK-STAT pathway, which subsequently promotes axon regeneration (57). This finding is consistent with the report by Sun et al (58). With regard to the different isoforms of JAK, it appears that JAK1 is predominantly associated with astrocytic differentiation (33) while JAK2 is considered to be essential for NSC proliferation $(59,60)$. Other studies have indicated that the deletion of JAK2 inhibits the activation of c-myc and c-fos promoters and cell proliferation (61); additionally, the absence of STAT5 suppresses the induction of c-fos and blocks cell cycle progression (62), confirming that JAK2 and STAT5 may be indispensable in cell proliferation. The silencing of JAK3 induces to the differentiation of NPC into neurons and oligodendrocytes (60). Furthermore, the suppression of STAT3 in vitro (63) or its conditional ablation in vivo (52) has been validated to promote neurogenesis.

\section{Time-dependent effects of the JAK-STAT pathway in reactive astrocytes}

Kernie et al (64) reported that a significant amount of the formation of astrogliotic scars after CNS trauma may be attributed to newly generated astrocytes, but not to the activation or migration of resident astrocytes. The traditional view is that reactive astrocytes can inhibit axonal regrowth due to the microenvironmental factors that significantly alter immediately following SCI. The major causes include production of chondroitin sulfate proteoglycans (65), a type 
of inhibitory extracellular matrix molecule, and the release of pro-inflammatory cytokines, including IL-1 $\beta$, IL-6 and tumor necrosis factor- $\alpha$ (TNF- $\alpha$ ) (66-69). These pro-inflammatory cytokines are immunoreactive for phosphorylated STAT3 following SCI; this indicates a role for the phosphorylation of STAT3 in the activation of astrocytes (70-72), particularly in chronic phases, within injury of the spinal cords. Okada et al (73) confirmed that suppression of the IL-6 receptor not only inhibited the astrocytic differentiation promoted by IL- 6 signaling via the JAK-STAT pathway, but also prevented the development of astrogliosis, which reduces the axonal regeneration in the chronic phases after SCI $(73,74)$. In a relative sense, a time-dependent analysis of the reactive astrocytes is critical for the identification of its effects in the injured spinal cord. At the acute and subacute phases after SCI, the reactive astrocytes serve to separate the healthy tissue from the lesion area by restoring the blood-spinal cord barrier (75). This prevents the potential overwhelming inflammatory response (76), massive cellular degeneration and death (77), and tissue damage during the secondary injury (78). Therefore, a number scholars believe that astrogliosis after CNS injury is dependent on STAT3 activation, an indispensable step for the formation of glia scar and limitation of the spread of inflammation (70). Additional studies reported that the deletion of STAT3 following SCI leads to the limited migration of reactive astrocytes, which was associated with widespread infiltration of inflammatory cells, demyelination and severe loss of motor function $(79,80)$. Furthermore, this finding was also validated by a study by Leung et al (81), where conditional ablation of SOCS3 was observed to prolong expression of STAT3 in reactive astrocytes and significantly improve wound healing and motor function.

\section{Astrocyte-secretory polypeptides promote neuroprotec- tion via activating the JAK-STAT pathway}

The neuroprotective effect of reactive astrocytes is multifaceted and includes astrocyte-secretory polypeptides, which contribute to endogenous neuroprotection and repair. Reactive astrocytes can secrete and respond to a number of vital cytokines, which affects the cellular state of the surrounding cells (microglia and neurons) and of astrocytes themselves (82). Reactive astrocytes can preserve neurons and oligodendrocytes, and protect motor functions after SCI $(72,76,78)$, potentially due to the astrocyte-secretory polypeptides (astrocyte-derived cytokines and trophic factors), which alter the microenvironment (83-85). These cytokines include IL-1 $\beta$, TNF- $\alpha$, IL-6, IL-11 and transforming growth factor- $\beta 1$ (86-92) and the trophic factors include brain-derived neurotrophic factor, glial cell line-derived neurotrophic factor, nerve growth factor (NGF), CNTF, basic fibroblast growth factor and leukemia inhibiting factor (LIF) (93-97). Recently, increasing evidence has indicated that cytokines and trophic factors secreted by reactive astrocytes may protect the injured tissues and cells through the JAK-STAT pathway $(82,98)$.

Specifically, IL-6 and its family members, such as IL-11, LIF and CNTF may be activators of JAK-STAT signalling in neurons following SCI (99-103). Yamauchi et al (104) demonstrated the peak expression of IL- 6 to be consistent with the maximum activation of JAK1 and STAT3 in neurons, with translocation of phosphorylated STAT3 to the nucleus. It has previously been validated that the co-administration of IL-6 and soluble IL-6 receptor can improve neurological manifestations and protect motor neurons of the spinal cord from degeneration (105). Additional research also indicated that, compared with wild-type mice after injury, IL-6 gene knockout mice exhibited more severe damage and death of the spinal cord neurons (106). Further studies have provided evidence that IL-6 is significant in the regulation of sensory functions in vivo (107). The study by Yamauchi et al (104) revealed that pretreatment with the JAK2 inhibitor, AG-490 (108) reduced the functional recovery of hindlimbs after SCI, which indicated that activation of the JAK-STAT pathway induced by IL-6 in neurons may contribute to neuroprotection after SCI. As an effective trophic factor and pro-inflammatory factor, the LIF concentration increased within $24 \mathrm{~h}$ following SCI, indicating its vital role in regulating inflammatory reactions and preservation of oligodendrocyte following SCI $(80,109)$. Although NGF and CNTF have diverse effects on the CNS, including differentiation and proliferation, they are able to enhance the survival of oligodendrocyte progenitors in CNS (110-117). Oyesiku et al (118) have previously indicated an upregulation of CNTF-receptors in the motoneurons of ventral horn and increase of CNTF in white matter, within $24 \mathrm{~h}$ after SCI. The expression of CNTF in reactive astrocytes was triggered by the SCI (119). Dell'Albani et al (120) reported that CNTF, induces a rapid tyrosine phosphorylation of JAK1, JAK2, STAT1a/b and STAT3. The authors also considered JAK-STATs to be crucial in enhancing cell survival in CNS.

The activation of STAT not only rapidly activates caspase-9, -7, -6 and -3 (121) but also induces the transcription of non-apoptotic proteins. It appears that the ratio of STAT1 and STAT3 or STAT5 activation is significant in cell survival and apoptosis, with STAT1 being more apoptotic and STAT3 and STAT5 exhibiting anti-apoptotic properties (122). Several studies have demonstrated that STAT3 phosphorylation is involved in neuroprotection. Cheng et al (22) reported that the activation of STAT3 may be associated with the reduction of neuronal apoptosis following cerebral injury. Suzuki et al (123) revealed that the rapid enhancement of phosphorylated STAT3 was detected after the application of high-dose recombinant LIF. The phosphorylation of STAT3 correlated with a downregulation of damage to the $\mathrm{CNS}$, including a decrease in the number of TUNEL-positive cells in the damage area $(123,124)$. Another study was also consistent with this finding; inhibition of STAT3 phosphorylation following damage to the CNS was associated with an increased seriousness of the secondary injury, and potential enlargement of the lesion, and exacerbation of neurological deficits (125). Neuroprotection requires the active suppression of apoptosis, which is accomplished either through suppressing caspases or by inhibiting their activation. The function of STAT3 in neuroprotection appears to be associated with the transcriptional modulation of antiapoptotic regulatory proteins, including the Bcl-2 family (126). Ahn et al (127) reported that survival factors cause the activation of STAT3, which was upregulated at $6 \mathrm{~h}$ following SCI; this prevents the release of cytochrome $c$ and subsequent activation of caspase through the induction of Bcl-2 and Bcl-xL. All of these factors indicate that the increased expression of the transcription factors of the JAK and STAT family have antiapoptotic effects, which promote neuronal protection. 


\section{Conclusions}

The JAK-STAT pathway is a critical pathway in proliferation and differentiation of NSCs and NPCs after SCI; it has been validated that the JAK-STAT pathway is significant in astrocytic differentiation, which is closely associated with glial scar formation and neuroprotection following SCI. Contrary to the conventional view that reactive astrocytes may inhibit axonal regrowth, the present view is that time-dependent effects of the JAK-STAT pathway in reactive astrocytes must be considered. In particular, that these effects are positive and protective in acute and subacute phases, while negative and inhibitory effects are observed in chronic phases. Reactive astrocytes may promote neuronal and oligodendrocytic protection and also protect motor functions following SCI. This is due to cytokines and trophic factors, including IL-6, IL-11, LIF and CNTF, secreted by reactive astrocytes. This indicates the neuronal protection and prevention of demyelination, partially through the JAT-STAT pathway. However, the mechanisms and the association between the JAK-STAT pathway, NSCs and NPCs, reactive astrocytes and astrocyte-secretory polypeptides remain unclear. Further studies are required to elucidate these mechanisms and associations to improve understanding of SCI and its treatment further detail. Elucidation of the mechanisms of JAK-STAT pathway may improve treatment with stem cell transplantation and aid in the identification of a new therapeutic tool that induces neuroprotection by controlling the function of reactive astrocytes.

\section{Acknowledgements}

This study was supported by the Medical Science and Technology Youth Cultivation Project of the Chinese People's Liberation Army (grant no. 13QNP017), the Key Program of National Natural Science Foundation of China (grant no. 81330042), the General Program of National Natural Science Foundation of China (grant nos. 81371957 and 81171714), the Special Project between Ministry of Science and Technology of China and Russia (grant no. 2014DFR31210) and the Cooperation Project between Tianjin Municipal Science and Technology Commission and Australia (grant no. 13RCGFSY19000).

\section{References}

1. Hua R, Shi J, Wang X, et al: Analysis of the causes and types of traumatic spinal cord injury based on 561 cases in China from 2001 to 2010. Spinal Cord 51: 218-221, 2013.

2. Cao HQ and Dong ED: An update on spinal cord injury research. Neurosci Bull 29: 94-102, 2013.

3. Pickelsimer E, Shiroma EJ and Wilson DA: Statewide investigation of medically attended adverse health conditions of persons with spinal cord injury. J Spinal Cord Med 33: 221-231, 2010

4. Huang H, Fan S, Ji X, Zhang Y, Bao F and Zhang G: Recombinant human erythropoietin protects against experimental spinal cord trauma injury by regulating expression of the proteins MKP-1 and p-ERK. J Int Med Res 37: 511-519, 2009.

5. Oyinbo CA: Secondary injury mechanisms in traumatic spinal cord injury: a nugget of this multiply cascade. Acta Neurobio Exp (Wars) 71: 281-299, 2011.

6. Nicolas CS, Amici M, Bortolotto ZA, et al: The role of JAK-STAT signaling within the CNS. JAKSTAT 2: e22925, 2013.

7. Laurence A, Pesu M, Silvennoinen O and O'Shea J: JAK kinases in health and disease: an update. Open Rheumatol J 6 : 232-244, 2012.

8. Aittomaki S and Pesu M: Therapeutic targeting of the Jak/STAT pathway. Basic Clin Pharmacol Toxicol 114: 18-23, 2014.
9. Darnell JE Jr, Kerr IM and Stark GR: Jak-STAT pathways and transcriptional activation in response to IFNs and other extracellular signaling proteins. Science 264: 1415-1421, 1994.

10. Shuai K, Horvath CM, Huang LH, Qureshi SA, Cowburn D and Darnell JE Jr: Interferon activation of the transcription factor Stat91 involves dimerization through $\mathrm{SH} 2$-phosphotyrosyl peptide interactions. Cell 76: 821-828, 1994.

11. O'Shea JJ, Holland SM and Staudt LM: JAKs and STATs in immunity, immunodeficiency, and cancer. N Engl J Med 368: 161-170, 2013.

12. O'Shea JJ and Plenge R: JAK and STAT signaling molecules in immunoregulation and immune-mediated disease. Immunity 36 : 542-550, 2012.

13. Lutticken C, Wegenka UM, Yuan J, Buschmann J, et al: Association of transcription factor APRF and protein kinase Jak1 with the interleukin-6 signal transducer gp130. Science 263: 89-92, 1994.

14. Stahl N, Boulton TG, Farruggella T, et al: Association and activation of Jak-Tyk kinases by CNTF-LIF-OSM-IL- 6 beta receptor components. Science 263: 92-95, 1994.

15. Sadowski HB, Shuai K, Darnell JE Jr and Gilman MZ: A common nuclear signal transduction pathway activated by growth factor and cytokine receptors. Science 261: 1739-1744, 1993.

16. Zhong Z, Wen Z and Darnell JE Jr: Stat3: a STAT family member activated by tyrosine phosphorylation in response to epidermal growth factor and interleukin-6. Science 264: 95-98, 1994.

17. Vahedi G, Takahashi H, Nakayamada S, et al: STATs shape the active enhancer landscape of T cell populations. Cell 151: 981-993, 2012.

18. Schwaiger FW, Hager G, Schmitt AB, et al: Peripheral but not central axotomy induces changes in Janus kinases (JAK) and signal transducers and activators of transcription (STAT). Eur J Neurosci 12: 1165-1176, 2000.

19. Yao GL, Kato H, Khalil M, Kiryu S and Kiyama H: Selective upregulation of cytokine receptor subchain and their intracellular signalling molecules after peripheral nerve injury. Eur J Neurosci 9: 1047-1054, 1997.

20. Choi JS, Kim SY, Cha JH, et al: Upregulation of gp130 and STAT3 activation in the rat hippocampus following transient forebrain ischemia. Glia 41: 237-246, 2003.

21. Suzuki S, Tanaka K, Nogawa S, Dembo T, Kosakai A and Fukuuchi Y: Phosphorylation of signal transducer and activator of transcription-3 (Stat3) after focal cerebral ischemia in rats. Exp Neurol 170: 63-71, 2001.

22. Wen TC, Peng H, Hata R, Desaki J and Sakanaka M: Induction of phosphorylated-Stat 3 following focal cerebral ischemia in mice. Neurosci Lett 303: 153-156, 2001.

23. Cheng Z, Li L, Mo X, et al: Non-invasive remote limb ischemic postconditioning protects rats against focal cerebral ischemia by upregulating STAT3 and reducing apoptosis. Int J Mol Med 34: 957-966, 2014.

24. Justicia C, Gabriel C and Planas AM: Activation of the JAK/STAT pathway following transient focal cerebral ischemia: signaling through Jak1 and Stat3 in astrocytes. Glia 30: 253-270, 2000.

25. Stromberg H, Svensson SP and Hermanson O: Distribution of the transcription factor signal transducer and activator of transcription 3 in the rat central nervous system and dorsal root ganglia. Brain Res 853: 105-114, 2000.

26. Johansson CB, Momma S, Clarke DL, Risling M, Lendahl U and Frisen J: Identification of a neural stem cell in the adult mammalian central nervous system. Cell 96: 25-34, 1999.

27. Takahashi M, Arai Y, Kurosawa H, Sueyoshi N and Shirai S: Ependymal cell reactions in spinal cord segments after compression injury in adult rat. J Neuropathol Exp Neurol 62: 185-194, 2003.

28. Bjorklund A and Lindvall O: Self-repair in the brain. Nature 405: 892-893, 895, 2000.

29. Horner PJ, Power AE, Kempermann G, et al: Proliferation and differentiation of progenitor cells throughout the intact adult rat spinal cord. J Neurosci 20: 2218-2228, 2000.

30. Lu P, Graham L, Wang Y, Wu D and Tuszynski M: Promotion of survival and differentiation of neural stem cells with fibrin and growth factor cocktails after severe spinal cord injury. J Vis Exp: 27: e50641, 2014

31. Tuszynski MH, Wang Y, Graham L, et al: Neural stem cells in models of spinal cord injury. Exp Neurol 261C: 494-500, 2014.

32. Natarajan R, Singal V, Benes R, et al: STAT3 modulation to enhance motor neuron differentiation in human neural stem cells. PLoS One 9: e100405, 2014. 
33. Xiao Q, Du Y, Wu W and Yip HK: Bone morphogenetic proteins mediate cellular response and, together with Noggin, regulate astrocyte differentiation after spinal cord injury. Exp Neurol 221: 353-366, 2010.

34. Bonni A, Sun Y, Nadal-Vicens M, et al: Regulation of gliogenesis in the central nervous system by the JAK-STAT signaling pathway. Science 278: 477-483, 1997.

35. He F, Ge W, Martinowich K, et al: A positive autoregulatory loop of Jak-STAT signaling controls the onset of astrogliogenesis. Nat Neurosci 8: 616-625, 2005.

36. Nakanishi M, Niidome T, Matsuda S, Akaike A, Kihara T and Sugimoto H: Microglia-derived interleukin-6 and leukaemia inhibitory factor promote astrocytic differentiation of neural stem/progenitor cells. Eur J Neurosci 25: 649-658, 2007.

37. Nakashima K, Yanagisawa M, Arakawa $\mathrm{H}$, et al: Synergistic signaling in fetal brain by STAT3-Smad1 complex bridged by p300. Science 284: 479-482, 1999.

38. Sriram K, Benkovic SA, Hebert MA, Miller DB and O'Callaghan JP: Induction of gp130-related cytokines and activation of JAK2/STAT3 pathway in astrocytes precedes up-regulation of glial fibrillary acidic protein in the 1-methyl-4-phenyl-1,2,3,6-tetrahydropyridine model of neurodegeneration: key signaling pathway for astrogliosis in vivo? J Biol Chem 279: 19936-19947, 2004

39. Taga T, Hibi M, Hirata Y, et al: Interleukin-6 triggers the association of its receptor with a possible signal transducer, gp130. Cell 58: 573-581, 1989.

40. Mangoura D, Pelletiere C, Leung S, Sakellaridis N and Wang DX: Prolactin concurrently activates src-PLD and JAK/Stat signaling pathways to induce proliferation while promoting differentiation in embryonic astrocytes. Int J Dev Neurosci 18: 693-704, 2000.

41. David M,Petricoin EF III, Igarashi K, Feldman GM,Finbloom DS and Larner AC: Prolactin activates the interferon-regulated p91 transcription factor and the Jak2 kinase by tyrosine phosphorylation. Proc Natl Acad Sci USA 91: 7174-7178, 1994

42. Dusanter-Fourt I, Muller O, Ziemiecki A, et al: Identification of JAK protein tyrosine kinases as signaling molecules for prolactin. Functional analysis of prolactin receptor and prolactin-erythropoietin receptor chimera expressed in lymphoid cells. EMBO J 13: 2583-2591, 1994

43. Gilmour KC and Reich NC: Receptor to nucleus signaling by prolactin and interleukin 2 via activation of latent DNA-binding factors. Proc Natl Acad Sci USA 91: 6850-6854, 1994.

44. Lebrun JJ, Ali S, Sofer L, Ullrich A and Kelly PA: Prolactin-induced proliferation of $\mathrm{Nb} 2$ cells involves tyrosine phosphorylation of the prolactin receptor and its associated tyrosine kinase JAK2. J Biol Chem 269: 14021-14026, 1994.

45. Rui H, Djeu JY, Evans GA, Kelly PA and Farrar WL: Prolactin receptor triggering. Evidence for rapid tyrosine kinase activation. J Biol Chem 267: 24076-24081, 1992.

46. Rui H, Kirken RA and Farrar WL: Activation of receptor-associated tyrosine kinase JAK2 by prolactin. J Biol Chem 269 5364-5368, 1994.

47. DeVito WJ, Avakian C, Stone S and Okulicz WC: Prolactin-stimulated mitogenesis of cultured astrocytes is mediated by a protein kinase C-dependent mechanism. J Neurochem 60: 832-842, 1993.

48. DeVito WJ, Avakian C, Stone S, Okulicz WC, Tang KT and Shamgochian M: Prolactin induced expression of interleukin-1 alpha, tumor necrosis factor-alpha, and transforming growth factor-alpha in cultured astrocytes. J Cell Biochem 57: 290-298, 1995.

49. DeVito WJ, Okulicz WC, Stone $\mathrm{S}$ and Avakian C: Prolactin-stimulated mitogenesis of cultured astrocytes Endocrinology 130: 2549-2556, 1992.

50. DeVito WJ, Stone S and Mori K: Low concentrations of ethanol inhibits prolactin-induced mitogenesis and cytokine expression in cultured astrocytes. Endocrinology 138: 922-928, 1997.

51. DeVito WJ, Stone S and Shamgochian M: Prolactin induced expression of glial fibrillary acidic protein and tumo necrosis factor-alpha at a wound site in the rat brain. Mol Cell Endocrinol 108: 125-130, 1995

52. DeVito WJ and Stone S: Ethanol inhibits prolactin-induced activation of the JAK/STAT pathway in cultured astrocytes. J Cell Biochem 74: 278-291, 1999.

53. Cao F, Hata R, Zhu P, Nakashiro K and Sakanaka M: Conditional deletion of Stat 3 promotes neurogenesis and inhibits astrogliogenesis in neural stem cells. Biochem Biophys Res Commun 394 843-847, 2010.

54. Bauer S: Cytokine control of adult neural stem cells. Ann NY Acad Sci 1153: 48-56, 2009.
55. Gomez-Nicola D, Valle-Argos B, Pallas-Bazarra N and Nieto-Sampedro M: Interleukin-15 regulates proliferation and self-renewal of adult neural stem cells. Mol Biol Cell 22: 1960-1970, 2011

56. Gupta S, Mishra K, Surolia A and Banerjee K: Suppressor of cytokine signalling-6 promotes neurite outgrowth via JAK2/STAT5-mediated signalling pathway, involving negative feedback inhibition. PLoS One 6: e26674, 2011.

57. Turnley AM, Faux CH, Rietze RL, Coonan JR and Bartlett PF: Suppressor of cytokine signaling 2 regulates neuronal differentiation by inhibiting growth hormone signaling. Nat Neurosci 5: $1155-1162,2002$

58. Smith PD, Sun F, Park KK, et al: SOCS3 deletion promotes optic nerve regeneration in vivo. Neuron 64: 617-623, 2009.

59. Sun F, Park KK, Belin S, et al: Sustained axon regeneration induced by co-deletion of PTEN and SOCS3. Nature 480: 372-375, 2011.

60. Garza JC, Guo M, Zhang W and Lu XY: Leptin increases adult hippocampal neurogenesis in vivo and in vitro. J Biol Chem 283 . 18238-18247, 2008.

61. Kim YH, Chung JI, Woo HG, et al: Differential regulation of proliferation and differentiation in neural precursor cells by the Jak pathway. Stem Cells 28: 1816-1828, 2010.

62. Watanabe $\mathrm{S}$, Itoh $\mathrm{T}$ and Arai K: Roles of JAK kinase in human GM-CSF receptor signals. Leukemia 11 (Suppl 3): 76-78, 1997.

63. Mui AL, Wakao H, Kinoshita T, Kitamura T and Miyajima A: Suppression of interleukin-3-induced gene expression by a $\mathrm{C}$-terminal truncated Stat5: role of Stat5 in proliferation. EMBO J 15: 2425-2433, 1996.

64. Gu F, Hata R, Ma YJ, et al: Suppression of Stat3 promotes neurogenesis in cultured neural stem cells. J Neurosci Res 81: 163-171, 2005.

65. Kernie SG, Erwin TM and Parada LF: Brain remodeling due to neuronal and astrocytic proliferation after controlled cortical injury in mice. J Neurosci Res 66: 317-326, 2001.

66. David S and Lacroix S: Molecular approaches to spinal cord repair. Annu Rev Neurosci 26: 411-440, 2003.

67. Bartholdi D and Schwab ME: Expression of pro-inflammatory cytokine and chemokine mRNA upon experimental spinal cord injury in mouse: an in situ hybridization study. Eur J Neurosci 9: $1422-1438,1997$.

68. Hayashi M, Ueyama T, Nemoto K, Tamaki T and Senba E: Sequential mRNA expression for immediate early genes, cytokines, and neurotrophins in spinal cord injury. J Neurotrauma 17: 203-218, 2000

69. Pan JZ, Ni L, Sodhi A, Aguanno A, Young W and Hart RP: Cytokine activity contributes to induction of inflammatory cytokine mRNAs in spinal cord following contusion. J Neurosci Res 68: 315-322, 2002.

70. Streit WJ, Semple-Rowland SL, Hurley SD, Miller RC, Popovich PG and Stokes BT: Cytokine mRNA profiles in contused spinal cord and axotomized facial nucleus suggest a beneficial role for inflammation and gliosis. Exp Neurol 152: 74-87, 1998

71. Herrmann JE, Imura T, Song B, et al: STAT3 is a critical regulator of astrogliosis and scar formation after spinal cord injury. J Neurosci 28: 7231-7243, 2008.

72. Satriotomo I, Bowen KK and Vemuganti R: JAK2 and STAT3 activation contributes to neuronal damage following transient focal cerebral ischemia. J Neurochem 98: 1353-1368, 2006.

73. Kang W and Hebert JM: Signaling pathways in reactive astrocytes, a genetic perspective. Mol Neurobiol 43: 147-154, 2011.

74. Okada S, Nakamura M,Mikami Y, et al: Blockade of interleukin-6 receptor suppresses reactive astrogliosis and ameliorates functional recovery in experimental spinal cord injury. J Neurosci Res 76: 265-276, 2004.

75. Rolls A, Shechter R and Schwartz M: The bright side of the glial scar in CNS repair. Nat Rev Neurosci 10: 235-241, 2009.

76. Yuan YM and He C: The glial scar in spinal cord injury and repair. Neurosci Bull 29: 421-435, 2013.

77. Karimi-Abdolrezaee S and Billakanti R: Reactive astrogliosis after spinal cord injury-beneficial and detrimental effects. Mol Neurobiol 46: 251-264, 2012.

78. Silver J and Miller JH: Regeneration beyond the glial scar. Nat Rev Neurosci 5: 146-156, 2004.

79. Faulkner JR, Herrmann JE, Woo MJ, Tansey KE, Doan NB and Sofroniew MV: Reactive astrocytes protect tissue and preserve function after spinal cord injury. J Neurosci 24: 2143-2155, 2004

80. Okada S, Nakamura M, Katoh H, et al: Conditional ablation of Stat3 or Socs3 discloses a dual role for reactive astrocytes after spinal cord injury. Nat Med 12: 829-834, 2006. 
81. Cho N, Nguyen DH, Satkunendrarajah K, Branch DR and Fehlings MG: Evaluating the role of IL-11, a novel cytokine in the IL-6 family, in a mouse model of spinal cord injury. J Neuroinflammation 9: 134, 2012.

82. Leung YK, Pankhurst M, Dunlop SA, et al: Metallothionein induces a regenerative reactive astrocyte phenotype via JAK/STAT and RhoA signalling pathways. Exp Neurol 221: 98-106, 2010.

83. Hamby ME and Sofroniew MV: Reactive astrocytes as therapeutic targets for CNS disorders. Neurotherapeutics 7: 494-506, 2010.

84. Lieberman AP, Pitha PM, Shin HS and Shin ML: Production of tumor necrosis factor and other cytokines by astrocytes stimulated with lipopolysaccharide or a neurotropic virus. Proc Natl Acad Sci USA 86: 6348-6352, 1989.

85. Sofroniew MV: Molecular dissection of reactive astrogliosis and glial scar formation. Trends Neurosci 32: 638-647, 2009.

86. Chu T, Zhou H, Li F, Wang T, Lu L and Feng S: Astrocyte transplantation for spinal cord injury: current status and perspective. Brain Res Bull 107C: 18-30, 2014.

87. Hamby ME, Hewett JA and Hewett SJ: TGF-betal reduces the heterogeneity of astrocytic cyclooxygenase-2 and nitric oxide synthase-2 gene expression in a stimulus-independent manner. Prostaglandins Other Lipid Mediat 85: 115-124, 2008.

88. Hamby ME, Gragnolati AR, Hewett SJ and Hewett JA: TGF beta 1 and TNF alpha potentiate nitric oxide production in astrocyte cultures by recruiting distinct subpopulations of cells to express NOS-2. Neurochem Int 52: 962-971, 2008.

89. Hamby ME, Hewett JA and Hewett SJ: TGF-beta1 potentiates astrocytic nitric oxide production by expanding the population of astrocytes that express NOS-2. Glia 54: 566-577, 2006.

90. Hewett SJ: Interferon-gamma reduces cyclooxygenase-2-mediated prostaglandin E2 production from primary mouse astrocytes independent of nitric oxide formation. J Neuroimmunol 94: 134-143, 1999.

91. Minghetti L, Polazzi E, Nicolini A and Levi G: Opposite regulation of prostaglandin E2 synthesis by transforming growth factor-betal and interleukin 10 in activated microglial cultures. J Neuroimmunol 82: 31-39, 1998.

92. Hewett SJ, Corbett JA, McDaniel ML and Choi DW: Interferon-gamma and interleukin-1 beta induce nitric oxide formation from primary mouse astrocytes. Neurosci Lett 164 229-232, 1993.

93.Zhang Y, Taveggia C, Melendez-Vasquez C, et al: Interleukin-11 potentiates oligodendrocyte survival and maturation, and myelin formation. J Neurosci 26: 12174-12185, 2006.

94. Mena MA and Garcia de Yebenes J: Glial cells as players in parkinsonism: the 'good,' the 'bad,' and the 'mysterious' glia Neuroscientist 14: 544-560, 2008

95. Villoslada P and Genain CP: Role of nerve growth factor and other trophic factors in brain inflammation. Prog Brain Res 146 403-414, 2004

96. Krieglstein K, Reuss B, Maysinger D and Unsicker K: Short communication: transforming growth factor-beta mediates the neurotrophic effect of fibroblast growth factor- 2 on midbrain dopaminergic neurons. Eur J Neurosci 10: 2746-2750, 1998.

97. Ridet JL, Malhotra SK, Privat A and Gage FH: Reactive astrocytes: cellular and molecular cues to biological function. Trends Neurosci 20: 570-577, 1997.

98. Saad B, Constam DB, Ortmann R, Moos M, Fontana A and Schachner M: Astrocyte-derived TGF-beta 2 and NGF differentially regulate neural recognition molecule expression by cultured astrocytes. J Cell Biol 115: 473-484, 1991.

99. White RE and Jakeman LB: Don't fence me in: harnessing the beneficial roles of astrocytes for spinal cord repair. Restor Neurol Neurosci 26: 197-214, 2008

100. Cattaneo E, Conti L and De-Fraja C: Signalling through the JAK-STAT pathway in the developing brain. Trends Neurosci 22: 365-369, 1999.

101. Heinrich PC, Behrmann I, Muller-Newen G, Schaper F and Graeve L: Interleukin-6-type cytokine signalling through the gp130/Jak/STAT pathway. Biochem J 334: 297-314, 1998.

102. Hirano T, Nakajima K and Hibi M: Signaling mechanisms through gp130: a model of the cytokine system. Cytokine Growth Factor Rev 8: 241-252, 1997.

103. Taga T: Gp130, a shared signal transducing receptor component for hematopoietic and neuropoietic cytokines. J Neurochem 67: $1-10,1996$.

104. Taga T and Kishimoto T: Gp130 and the interleukin-6 family of cytokines. Annu Rev Immunol 15: 797-819, 1997.

105. Yamauchi K, Osuka K, Takayasu M, et al: Activation of JAK/STAT signalling in neurons following spinal cord injury in mice. J Neurochem 96: 1060-1070, 2006.
106. Ikeda K, Kinoshita M, Tagaya N, et al: Coadministration of interleukin-6 (IL-6) and soluble IL-6 receptor delays progression of wobbler mouse motor neuron disease. Brain Res 726: 91-97, 1996.

107. Pavelko KD, Howe CL, Drescher KM, et al: Interleukin-6 protects anterior horn neurons from lethal virus-induced injury. J Neurosci 23: 481-492, 2003.

108. Zhong J, Dietzel ID, Wahle P, Kopf M and Heumann R: Sensory impairments and delayed regeneration of sensory axons in interleukin-6-deficient mice. J Neurosci 19: 4305-4313, 1999.

109. Park JS, Lee J, Lim MA, et al: JAK2-STAT3 blockade by AG490 suppresses autoimmune arthritis in mice via reciprocal regulation of regulatory T cells and Th17 cells. J Immunol 192: 4417-4424, 2014.

110. Kerr BJ and Patterson PH: Potent pro-inflammatory actions of leukemia inhibitory factor in the spinal cord of the adult mouse. Exp Neurol 188: 391-407, 2004.

111. Barres BA, Hart IK, Coles HS, et al: Cell death and control of cell survival in the oligodendrocyte lineage. Cell 70: 31-46, 1992.

112. Barres BA, Schmid R, Sendnter M and Raff MC: Multiple extracellular signals are required for long-term oligodendrocyte survival. Development 118: 283-295, 1993.

113. D'Souza SD, Alinauskas KA and Antel JP: Ciliary neurotrophic factor selectively protects human oligodendrocytes from tumor necrosis factor-mediated injury. J Neurosci Res 43: 289-298, 1996

114. Kahn MA and De Vellis J: Regulation of an oligodendrocyte progenitor cell line by the interleukin- 6 family of cytokines. Glia 12: 87-98, 1994.

115. Louis JC, Magal E, Takayama S and Varon S: CNTF protection of oligodendrocytes against natural and tumor necrosis factor-induced death. Science 259: 689-692, 1993.

116. Noble M, Murray K, Stroobant P, Waterfield MD and Riddle P: Platelet-derived growth factor promotes division and motility and inhibits premature differentiation of the oligodendrocyte/type- 2 astrocyte progenitor cell. Nature 333: 560-562, 1988.

117. Raff MC, Lillien LE, Richardson WD, Burne JF and Noble MD: Platelet-derived growth factor from astrocytes drives the clock that times oligodendrocyte development in culture. Nature 333: $562-565,1988$

118. Richardson WD, Pringle N, Mosley MJ, Westermark B and Dubois-Dalcq M: A role for platelet-derived growth factor in normal gliogenesis in the central nervous system. Cell 53: 309-319, 1988 .

119. Oyesiku NM, Wilcox JN and Wigston DJ: Changes in expression of ciliary neurotrophic factor (CNTF) and CNTF-receptor alpha after spinal cord injury. J Neurobiol 32: 251-261, 1997.

120. Lee MY, Kim CJ, Shin SL, Moon SH and Chun MH: Increased ciliary neurotrophic factor expression in reactive astrocytes following spinal cord injury in the rat. Neurosci Lett 255: 79-82, 1998.

121.Dell'Albani P, Kahn MA, Cole R, Condorelli DF, Giuffrida-Stella AM and de Vellis J: Oligodendroglial survival factors, PDGF-AA and CNTF, activate similar JAK/STAT signaling pathways. J Neurosci Res 54: 191-205, 1998.

122. Dedoni S, Olianas MC and Onali P: Interferon-beta induces apoptosis in human SH-SY5Y neuroblastoma cells through activation of JAK-STAT signaling and down-regulation of PI3K/ Akt pathway. Journal of neurochemistry 115: 1421-1433, 2010.

123. Schindler C, Levy DE and Decker T: JAK-STAT signaling: from interferons to cytokines. J Biol Chem 282: 20059-20063, 2007.

124. Suzuki S, Tanaka K and Suzuki N: Ambivalent aspects of interleukin-6 in cerebral ischemia: inflammatory versus neurotrophic aspects. J Cereb Blood Flow Metab 29: 464-479, 2009.

125. Suzuki S, Yamashita T, Tanaka K, et al: Activation of cytokine signaling through leukemia inhibitory factor receptor (LIFR)/gp130 attenuates ischemic brain injury in rats. J Cereb Blood Flow Metab 25: 685-693, 2005.

126. Yamashita T, Sawamoto K, Suzuki S, et al: Blockade of interleukin- 6 signaling aggravates ischemic cerebral damage in mice: possible involvement of Stat 3 activation in the protection of neurons. J Neurochem 94: 459-468, 2005.

127. Battle TE and Frank DA: The role of STATs in apoptosis. Curr Mol Med 2: 381-392, 2002

128. Ahn YH, Lee G and Kang SK: Molecular insights of the injured lesions of rat spinal cords: inflammation, apoptosis, and cell survival. Biochem Biophys Res Commun 348: 560-570, 2006. 